\title{
Krüppel-like factor 8 involved in hypoxia promotes the invasion and metastasis of gastric cancer via epithelial to mesenchymal transition
}

\author{
NA LIU $^{1 *}$, YAFANG WANG $^{1 *}$, YONGAN ZHOU $^{2}$, HAILIN PANG $^{1}$, JING ZHOU $^{1}$, \\ PEI QIAN ${ }^{1}$, LILI LIU $^{1}$ and HELONG ZHANG ${ }^{1}$ \\ Departments of ${ }^{1}$ Oncology and ${ }^{2}$ Thoracic Surgery, Tangdu Hospital, Fourth Military Medical University, \\ Xi'an, Shaanxi 710038, P.R. China
}

Received April 14, 2014; Accepted July 17, 2014

DOI: 10.3892/or.2014.3495

\begin{abstract}
Previously, we reported that hypoxia was able to induce invasion and metastasis in gastric cancer and that hypoxia-inducible factor-1 (HIF-1) is a key factor involved in this tumor type. Krüppel-like factor 8 (KLF8) as a transcriptional repressor has been suggested as a promoter of tumor metastasis in breast cancer and an inducer of the epithelial-mesenchymal transition (EMT). KLF8 is also highly expressed in gastric cancer tissues, contributing to poor prognosis. However, the association between KLF8 and HIF-1 in regulating the progression of human gastric cancer in hypoxia is unclear. In the present study, we found that KLF8 was overexpressed in gastric cancer metastatic tissues and cells. Additionally, KLF8 siRNA significantly inhibited SGC7901 cell invasion and migration compared with SGC7901, SGC7901/Scr-si cells. Hypoxia is thus able to induce KLF8 expression and EMT in SGC7901 cells. However, following the examination of changes in cell morphology and epithelial and mesenchymal markers, it was found that KLF8 siRNA and HIF-1 siRNA strongly reversed EMT in cells undergoing hypoxia. Furthermore, hypoxia-induced KLF8 overexpression was attenuated by HIF-1 siRNA. Experiments using luciferase promoter constructs resulted in a marked increase in the activity of cells exposed to hypoxia and decreased activity in cells co-transfected with HIF-1 siRNA. The chromatin immunoprecipitation assay revealed proximal HRE at -133 is the main HIF-1 binding site in the KLF8 promoter. In conclusion, the results demonstrated that KLF8 is actively
\end{abstract}

Correspondence to: Professor Helong Zhang or Professor Lili Liu, Department of Oncology, Tangdu Hospital, Fourth Military Medical University, 1 Xinsi Road, Xi'an, Shaanxi 710038, P.R. China

E-mail: cnxazh1@163.com

E-mail: lily123fmmu@163.com

${ }^{*}$ Contributed equally

Key words: KLF8, hypoxia, gastric cancer, invasion, EMT enhanced by hypoxia and is a novel HIF-1 target. KLF8 is a novel EMT regulating transcription factor that involved in the progression of gastric cancer. The specific anti-EMT drugs in combination with anti-hypoxia are new promising cancer therapies.

\section{Introduction}

Tumor formation, evolution and metastasis are affected by oncogene, tumor-suppressor gene and cell cycle-related molecular. The regulation of transcription factors on potential downstream target genes plays an important role in the occurrence and progression of tumor (1). Hypoxia is the main factor for tumor migration and invasion, and hypoxia-inducible factor-1 (HIF-1) is involved in the processes $(2,3)$. In a previous study, we showed that hypoxia can induce gastric cancer invasion and migration (4), and HIF-1 is a key molecular regulator for the hypoxic signaling pathway (5).

HIF-1 upregulation is an early event of cells in hypoxia that triggers hypoxia-related gene transcription (6). HIF-1 can promote tumor angiogenesis, drug resistance and cell migration by binding with downstream $\operatorname{HRE}(7,8)$. The exact mechanism involved in HIF-1 promotion of gastric cancer metastasis under hypoxic conditions remains to be determined.

Under hypoxic conditions, a tumor microenvironment is generated and tumor cells undergo epithelial-to-mesenchymal transition (EMT) (9). During this process, tumor cells can adjust to the newly formed microenvironment and gain stem-cell characteristics that promote differentiation and proliferation of tumor cells. The continuous proliferation of tumor cells results in the center portion of hypoxia, which greatly promotes EMT $(10,11)$. The results of a previous study demonstrated that KLF8 was highly expressed in gastric cancer tissues and exhibited a poor prognosis (12). KLF8 was initially identified as a transcription repressor of Krüppel-like $\mathrm{C} 2 \mathrm{H} 2$ zinc-finger transcription factor family proteins (13). KLF8 influenced other tumor metastasis by EMT process (14) and was found to play an active role in renal cell carcinoma in which HIF-1 was highly expressed (15). However, the exact mechanism of KLF8 in hypoxia-induced EMT in gastric cancer remains to be elucidated. 
In the present study, we confirmed that KLF8 is important in gastric cancer invasion and metastasis. KLF8 siRNA-transfected SGC7901 cells showed less invasion and metastasis. Subsequently, HIF-1 siRNA was used to examine the effect of KLF8 expression and the mechanism of KLF8-induced EMT under hypoxia. We also confirmed that the HIF-1 binding site was located in the KLF8 promoter.

\section{Materials and methods}

Cell lines, cell culture conditions and hypoxic treatment. The SGC7901 human gastric adenocarcinoma cell line was obtained from the Academy of Military Medical Science (Beijing, China). The MKN45 and AGS human gastric adenocarcinoma cell lines were obtained from the Shanghai Cell Bank (Shanghai, China). The cell lines were cultured in RPMI-1640 medium containing 10\% fetal bovine serum (FBS) plus L-glutamine, vimentin, sodium pyruvate, non-essential amino acids, $100 \mathrm{IU} / \mathrm{ml}$ penicillin and $100 \mu \mathrm{g} / \mathrm{ml}$ streptomycin (Sigma-Aldrich, St. Louis, MO, USA). For hypoxic culture, the tumor cells were incubated in a hypoxic incubator (Precision Scientific, Winchester, VA, USA) with $1 \%$ atmospheric oxygen balanced by nitrogen and $\mathrm{CO}_{2}$ for $0-24 \mathrm{~h}$.

HIF-1 $\alpha$ siRNA plasmid constructs and transfection. The PSilencerTM neo U6 2.1 vector, containing a HIF-1 $\alpha$-specific targeting sequence (5'-AAAGAGGTGGATATGTCTGGG-3' and 5'-TTTCTCCACCTATACAGACCC-3') and a scramble sequence were introduced into SGC7901 cells. To observe the change in HIF-1 target genes induced by HIF-1 in hypoxia, we introduced the HIF-1 siRNA vector pSilenser3.1/HIF-si and control scramble siRNA vector pSilenser into SGC7901 cells, designated as SGC7901/HIF-si and SGC7901/Scr-si, respectively. The cells were transfected by using Lipofectamine 2000 (Invitrogen Life Technologies, Grand Island, NY, USA) according to the manufacturer's instructions and then treated with $0.4 \mathrm{mg} / \mathrm{ml}$ of G418 to select the transfected cells. Stably transfected cell clones were obtained by limiting the dilution culture under the pressure of G418. To avoid the effects of clonal variety, three random clones for each group were used in all the experiments.

KLF8 cDNA vector and RNAi lentivirus generation. KLF8-targeting oligonucleotides for generating cDNA were designed from full-length KLF8 by Shanghai GeneChem Co., Ltd. (Shanghai, China): forward, TCTGCAGGGACTACA GCAAG and reverse, TCACATTGGTGAATCCGTCT; KLF8 siRNA1 forward: ACUUGGAGGUCCAACUUAATT and reverse, UUAAGUUACCUCCAAGGTG; siRNA2 forward, CGAUAUGGAUAACUCAUATT and reverse, UAU GAGUUUAUCCAUAUCGAC; siRNA 3 forward, CACUGG UUAAUGACAUCAATT and reverse, UUGAUGUCAUUA AACAGUGCTA. After testing the overexpression and knockdown efficiencies, stem-loop oligonucleotides were synthesized and cloned into the lentivirus-based vector PsicoR (Addgene). A non-targeting stem-loop DNA PsicoR vector was generated as a negative control. Lentiviral particles were prepared as previously described (12). Gastric cancer cells were infected with KLF8 cDNA, siRNA-lentivirus or negative control virus at 7 days and examined at 10 days. The SGC7901 gastric cancer cells were transfected with KLF8 si1, KLF8 si2, KLF8 si3 or empty vector and designated as SGC7901/KLF-si1, SGC7901/ KLF-si2, SGC7901/ KLF-si3 and SGC7901/con. SGC7901 gastric cancer cells transfected with KLF8 vector or empty vector were designated as SGC7901/KLF and SGC7901/ con.

Cell migration assay. Transwell chamber polycarbonate membranes were used to determine the cell migration. SGC7901 cells $\left(5 \times 10^{4} /\right.$ well) were seeded on the top surface of the 24-well membrane with an $8-\mu \mathrm{m}$ pore culture insert (Millipore, Billerica, MA, USA). RPMI-1640 medium $(0.5 \mathrm{ml})$ plus $10 \%$ FBS was added to the bottom chamber as a chemoattractant. After $24 \mathrm{~h}$ incubation, the filter membrane was fixed with $4 \%$ paraformaldehyde and stained with hematoxylin and eosin. Non-invading cells on the surface inside the chamber were wiped off using a cotton swab. The number of migrated cells was counted by a microscope at 10 random fields of vision for each chamber. Experiments were performed in triplicate.

Cell invasion assay. The invasive activity of the SGC7901 cells was measured using 24-well BioCoat Matrigel Invasion Chambers (Millipore). In brief, the chamber containing the $8-\mu \mathrm{m}$ pore size filter was coated with Matrigel protein (BD Biosciences, Franklin Lakes, NJ, USA). The filters were subsequently inserted into a 24 -well culture plate. The SGC7901 cells were transfected with KLF siRNA or scramble siRNA for $\sim 48 \mathrm{~h}$ and then the cell density was adjusted to $\sim 5 \times 10^{4}$. The SGC7901 cell suspension was inoculated into each insert well and incubated with RPMI-1640 medium containing $10 \% \mathrm{FBS}$ for $24 \mathrm{~h}$ in $5 \% \mathrm{CO}_{2}$ at $37^{\circ} \mathrm{C}$. After $24 \mathrm{~h}$ of incubation, the non-invading cells on the upper surface inside the chamber were wiped off using a cotton swab. The invading cells on the lower surface of the chamber were stained with $0.2 \%$ crystal violet for $2 \mathrm{~h}$. The number of cells adhering to the lower surface of the filter was counted using a microcope at 10 random fields of vision for each chamber. Experiments were performed in triplicate.

Metastasis in nude mice. Mice were handled using best humane practices, and cared for in accordance with the NIH Animal Care and Use Committee guidelines. The cells were harvested from tissue culture flasks using trypsin and washed three times with PBS. The mice were injected through the tail vein with $1 \times 10^{6}$ cells in $0.1 \mathrm{ml}$ PBS, and monitored for overall health, and total body weight. Mice-bearing luciferasepositive tumors were imaged 4-6 weeks after injection with an IVIS Imaging System (Caliper Life Sciences, MA, USA). The mice were subsequently sacrificed and each experimental group comprised 5 mice.

Western blotting. The expression of KLF8, E-cadherin and fibronectin in SGC7901 cells transfected with KLF-siRNA or Scr-siRNA under normoxia conditions, as well as that of HIF-1, KLF8, E-cadherin, keratin, fibronectin and vimentin in SGC7901 cells transfected with KLF-siRNA, Scr-siRNA or HIF-siRNA under hypoxic conditions (1\% atmospheric oxygen) between 0 and $24 \mathrm{~h}$ was examined. Following pre-treatment, whole cells (SGC7901, SGC7901/KLF-si, SGC7901/Scr-si, SGC7901/HIF-si) were collected, respectively, and lysed on 
ice for $30 \mathrm{~min}$ in lysis buffer [10 mM Tris ( $\mathrm{pH} 8.0), 1 \mathrm{mM}$ ethylenediaminetetraacetic acid (EDTA), $400 \mathrm{mM} \mathrm{NaCl}$, $10 \%$ glycerol, $0.5 \%$ NP-40, $5 \mathrm{mM}$ sodium fluoride, $0.1 \mathrm{mM}$ phenylmethylsulfonyl fluoride, $1 \mathrm{mM}$ dithiothreitol]. Equal amounts of protein $(25 \mu \mathrm{g})$ were loaded. For western blotting, mouse monoclonal antibodies against KLF8 (1:200; Santa Cruz Biotechnology Inc., Santa Cruz, CA, USA), E-cadherin (1:2,500; Abcam, Cambridge, UK), fibronectin (1:2,500; Abcam), keratin (1:2,500; Abcam), vimentin (1:1,000; Abcam), anti-HIF-1 (mouse mAb; 1:200; Chemicon Corporation Ltd., Bangladesh), anti- $\beta$-actin (1:5,000; Sigma-Aldrich), anti-mouse secondary antibody (Ab; 1:2,000; Sigma-Aldrich), anti-rabbit secondary antibody (Ab; 1:2,000; Sigma-Aldrich). The relative content of each protein was detected by enhanced chemiluminescence (ECL; Amersham, Arlington Heights, IL, USA).

Real-time RT-PCR.The whole cells (SGC7901,SGC7901/Scr-si and SGC7901/HIF-si) were seeded after 0, 4, 8, 12 and $24 \mathrm{~h}$ exposure to hypoxia ( $1 \%$ atmospheric oxygen), respectively. RNA was isolated using RNAzol (Invitrogen, Carlsbad, CA, USA) according to the manufacturer's instructions. RNA $(1 \mu \mathrm{g})$ was subjected to reverse transcription, followed by cDNA strand synthesis at a dilution of 1:20. cDNA (10 $\mu \mathrm{l})$ was used for TaqMan real-time RT-PCR to detect mRNA expression. The ABI Prism 7700 Sequence Detection System (PE Applied Biosystems) was subsequently applied. The primers used were: KLF8 forward, 5'-TCTGCAGGGACTACAGCAAG and reverse, 5'-TCACATTGGTGAATCCGTCT; and GAPDH forward, 5'-TGGTATCGTGGAAGGACTCA and reverse, 5'-CCAGTAGAGGCAGGGATGAT. Experiments were performed in triplicate.

Immunofluorescence. SGC7901 cells were grown on 10-well glass microscope slides (VWR International, Fontenay-sousBois, France) under normoxic conditions, while SGC7901/ Scr-si, SGC7901/KLF-si cells were grown on 10-well glass microscope slides under hypoxic conditions (1\% atmospheric oxygen). The cells were fixed with $4 \%$ neutral formalin for 20 min and then permeabilized with $0.2 \%$ Triton $\mathrm{X}-100$ in PBS for $10 \mathrm{~min}$. After blocking with 3\% bovine serum albumin, the cells were incubated with E-cadherin and fibronectin primary antibody (BD Biosciences) overnight at $4^{\circ} \mathrm{C}$. After rinsing three times in PBS, the cells were incubated with a secondary antibody at room temperature. The nuclei were stained by DAPI (Roche Applied Science, Basel, Switzerland). Immunostaining signal and DAPI-stained nuclei were analyzed using a fluorescence microscope.

HIF-1 binding site search. A genomic region of 2,000 bp upstream of the KLF8 transcriptional initiation site was determined using the NCBI genomic BLAST program. The DNA strider 1.0 program was then applied to identify putative HRE. The HIF-1-binding consensus sequence BDCGTV (B is C/T/G; $\mathrm{D}$ is $\mathrm{A} / \mathrm{G} / \mathrm{T}$; and $\mathrm{V}$ is $\mathrm{G} / \mathrm{C} / \mathrm{A}$ ) was used as the key criterion for searching functional HRE. It was established using definitions of the consensus sequences, as previously provided (16).

Dual luciferase reporter gene assay. To assay the transcriptional activity of KLF8 under hypoxic conditions, SGC7901 cells in 24 -well plates $(50,000$ cells/well) were co-trans- fected with or without HIF-1 siRNA1 or KLF-Luc using Lipofectamine 2000 (Invitrogen). PRL-TK was the control for transfection efficiency in DMEM without serum. The construct KLF-Luc contained HRE-like or mutated sites from the KLF8 promoter nucleotides -133 to -128 , as 520 bp containing CTCGTG to CTTTTG, was synthesized and inserted into the pGL3 promoter vector (Promega, Madison, WI, USA). After $48 \mathrm{~h}$, luciferase activity was measured and quantified in a luminometer using the Dual-Luciferase Reporter Assay System (Promega). Experiments were performed in triplicate. Results are expressed as a means of the ratio between the firefly and Renilla luciferase activity.

Chromatin immunoprecipitation assay. HIF-1 binding to KLF8 promoter was analyzed by ChIP in gastric cancer cells, using methodologies previously described (5). Twenty-six SGC7901 cells were fixed with $1 \%$ paraformaldehyde, and chromatin derived from isolated nuclei was sheared by using a F550 microtip cell sonicator (Fisher Scientific, Morris Plains, NJ, USA). Following centrifugation, supernatants containing sheared chromatin were incubated with an anti-HIF-1 $\alpha$ antibody or control IgG. Protein A-Sepharose was added, the incubation was continued overnight, and immune complexes were subsequently eluted. The complexes were treated with RNase and proteinase $\mathrm{K}$ and extracted with phenol/chloroform and then with chloroform. DNA was precipitated, washed, dried, resuspended in water and analyzed by PCR. The primers used in this analysis spanned $216 \mathrm{bp}$ around the first possible HIF-1 $\alpha$ binding site located -133 bp from the translation start site (sense, 5'-GCAGGTACAAGGGCTGGGTA-3' and antisense, 5'-TGCCGGGACTGGGCTTTT-3').

Statistical analysis. Results were presented as means \pm SEM of at least three independent experiments. Bands from western blotting or RT-PCR analysis were quantified using Quantity One software (Bio-Rad, Hercules, CA, USA). Relative protein or mRNA levels were calculated relative to the amount of $\beta$-actin or GAPDH, respectively. The difference between means was performed with one-way analysis of variance or an unpaired t-test. All statistical analyses were performed using SPSS 13.0 software (SPSS, Chicago, IL, USA). P<0.05 was considered to indicate a statistically significant result.

\section{Results}

KLF8 is highly expressed in metastatic gastric cancer tissues and cells. The expression of KLF8 protein in non-metastatic and metastatic gastric cancer tissues was analyzed by western blotting. Four tissue samples were obtained from different primary sites. As shown in Fig. 1A, KLF8 protein expression was significantly upregulated in four primary sites of metastatic gastric cancer tissue compared with the non-metastatic gastric cancer tissue. RT-PCR was also performed to examine the KLF8 mRNA level of four different primary site tissues in non-metastatic gastric cancer and metastatic gastric cancer. Fig. 1B shows that KLF8 mRNA levels in metastatic gastric cancer tissue samples were significantly higher than the non-metastatic gastric cancer samples $(\mathrm{P}<0.05)$.

KLF8 protein and mRNA were examined in five types of gastric tumor cells of high and low metastatic potential. 

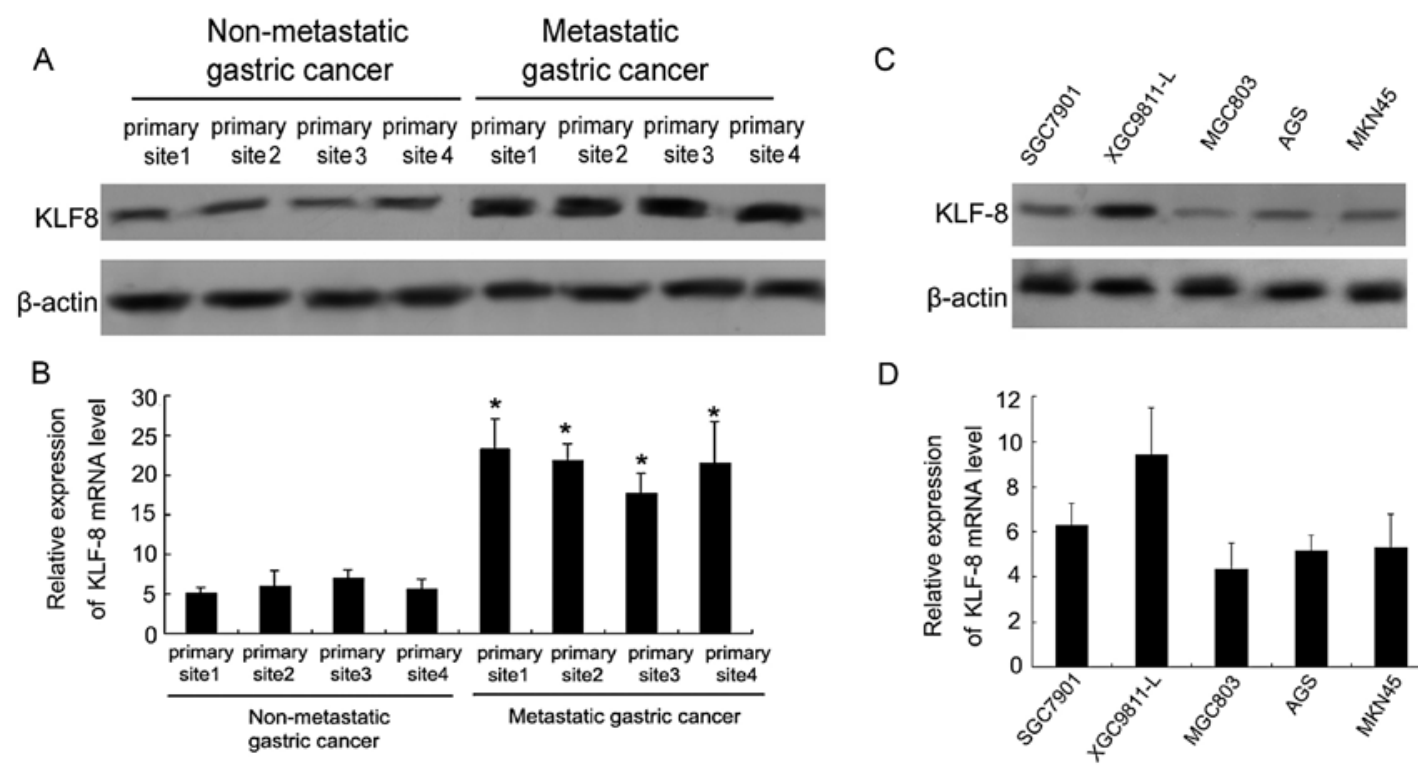

D

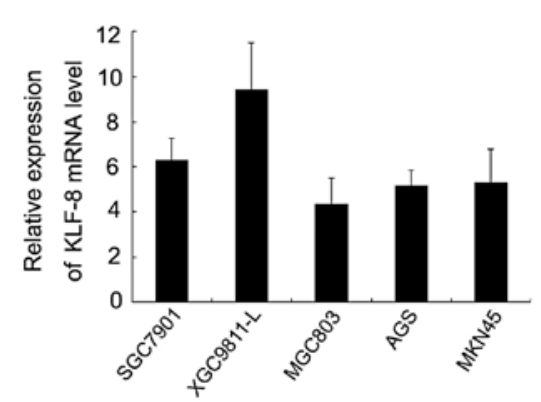

Figure 1. Krüppel-like factor 8 (KLF8) is highly increased in metastatic gastric cancer tissue and tumor cells. (A) Western blot analysis of KLF8 in non-metastatic and metastatic gastric cancer tissue, with the latter showing significant upregulation. (B) Analysis of KLF8 mRNA level normalized by GAPDH of non-metastatic and metastatic gastric cancer tissue. (C) Western blot analysis of KLF8 in in five types of gastric tumor cells of high and low metastatic potential. (D) RT-PCR analysis of KLF8 in five types of gastric tumor cells of high and low metastatic potential. "P $<0.05$ vs. non-metastatic gastric cancer.

XGC9811-L gastric tumor cells were found to have higher metastatic potential than the remaining four gastric tumor cells. A significantly higher expression level of KLF8 protein and mRNA in XGC9811-L cells is shown in Fig. 1C and D. The low metastatic potential cancer cells such as SGC7901, MGC803, AGS and MKN45 exhibited a lower expression of KLF8 protein and mRNA compared with XGC9811-L cells.

Forced overexpression of KLF8 promotes gastric tumor cell metastasis and induces EMT. To evaluate the role of KLF8 in the promotion of the invasion and migration in gastric cancer cells, a lentivirus-based delivery system was used to transfer the KLF8 sense vector and three siRNAs plasmids targeting KLF8 into SGC7901 cells. As shown in Fig. 2A, SGC7901 cells infected with KLF8 sense vector upregulated KLF8 expression. KLF8 siRNA1 markedly decreased KLF8 expression, while the effect of KLF-si3 was minimal. As shown in Fig. 2B, for each x200 field under inverted light microscope, the invasion cells were $148 \pm 13,186 \pm 25,127 \pm 14,125 \pm 10$, $86 \pm 7$ of SGC7901, SGC7901/KLF, SGC7901/con, SGC7901/ KLF-scr and SGC7901/KLF-si1 cells, respectively, suggesting that KLF8 siRNA1 significantly decreased the invasion ability of gastric cancer.

To determine the role of KLF8 in tumor growth and metastasis in vivo, SGC7901/KLF and SGC7901/con cells were administered into nude mice by the tail vein and flank region injection. Compared with the SGC7901/con group, the injection of SGC7901/KLF led to a significant increase in metastatic lesions (Fig. 2C). These results indicated that KLF8 overexpression had the potential to promote the invasion, migration and metastatic ability of SGC7901 cells in vitro and in vivo.

Morphological assessment of SGC7901 cells following infection with KLF8 cDNA indicated altered cell structure (Fig. 2D). Normal SGC7901 cells as control were gathered and polyhedral-shaped, whereas SGC7901-KLF8 appeared more scattered and spindle-shaped.

The protein expression of epithelial markers E-cadherin and keratin, and mesenchymal markers fibronectin and vimentin in SGC7901 cells transfected with KLF8 or negative control vector were analyzed by western blotting. As shown in Fig. 2E, SGC7901 cells transfected with KLF8 cDNA vector significantly downregulated E-cadherin and keratin expression and upregulated fibronectin and vimentin. These results suggested that KLF8 overexpression increased the invasion ability and induced EMT in gastric cancer.

Hypoxia induces KLF8 expression and KLF8 siRNA reversed hypoxia-induced EMT. SGC7901 gastric tumor cells were cultured in RPMI-1640 medium containing 10\% FBS for 0-24 h under hypoxia and the KLF8 and HIF-1 protein level were subsequently determined by western blot analysis. The KLF8 and HIF-1 expression increased under hypoxic condition in a time-dependent manner (Fig. 3A). RT-PCR was performed to examine the mechanism by which the KLF8 mRNA was upregulated by hypoxia. Fig. 3B shows that SGC7901 gastric tumor cells exposed to $1 \%$ oxygen condition induced a gradually increased expression of KLF8 mRNA in a time-dependent manner (means \pm SEM, $n=3$ ). These results led to investigation of the possibility of hypoxia-mediated transcriptional activation.

The morphological assessment of SGC7901 cells after $24 \mathrm{~h}$ transfection with siRNA against KLF8 under hypoxia indicated altered cell structure (Fig. 3D). Normal SGC7901 cells in normoxia culture appreared polyhedral-shaped. However, $24 \mathrm{~h}$ after KLF8 siRNA transfection, these cells appeared polyhedral and more gathered under hypoxia for $24 \mathrm{~h}$. The scramble siRNA produced a profound morphological change from polyhedral to fusiform. To investigate the mechanism of KLF8-induced EMT, we analyzed the 


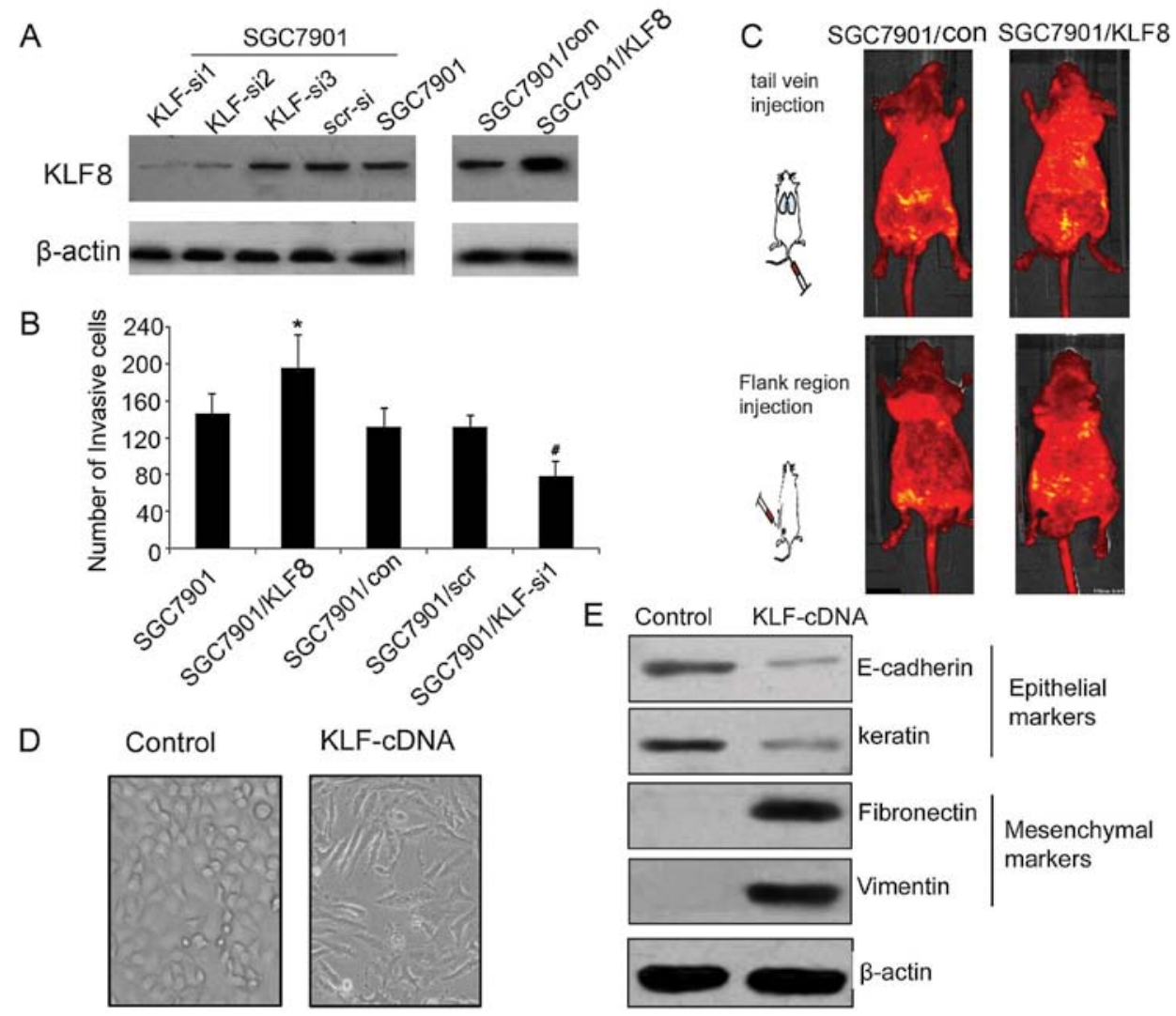

Figure 2. Overexpression of KLF8 promotes gastric tumor cell metastasis. (A) Western blot analysis of KLF8 protein expression in SGC7901 cells infected with the KLF8 sense vector and KLF8 siRNA. Actin was used as a loading control in this and other images. (B) Invasive ability was evaluated by counting cells invading through Matrigel and $8-\mu$ m pore Transwell membranes. ${ }^{*} \mathrm{P}<0.05$ vs. SGC7901/pc; ${ }^{\prime \prime} \mathrm{P}<0.05$ vs. SGC7901/scr. (C) Mice were injected with $1 \times 10^{6}$ cells through the tail vein and sacrificed after 4 weeks and using an IVIS Imaging System. (D) SGC7901 cells overexpressing KLF8 show spindle-like, fibroblastic morphology (magnification, x200). (E) Western blotting of KLF8 forced overexpression in SGC7901 cells using antibodies shows the loss of epithelial markers E-cadherin and keratin and the increase of mesenchymal markers fibronectin and vimentin. KLF8, Krüppel-like factor 8.
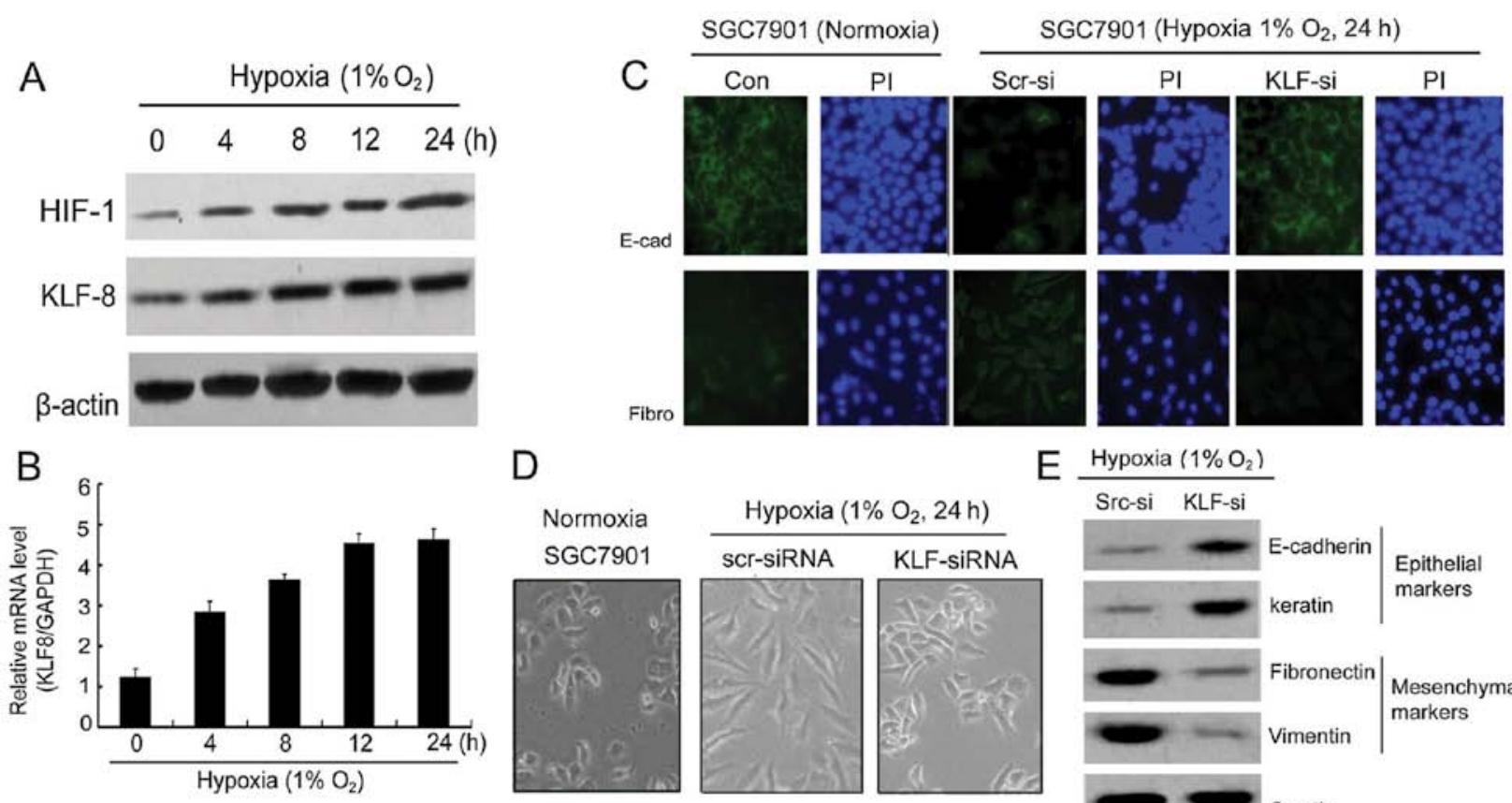

D
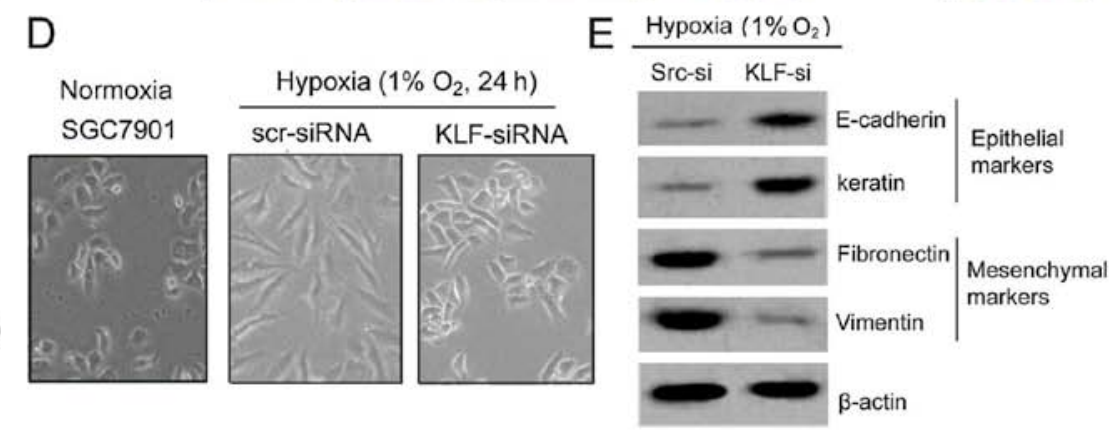

Figure 3. Hypoxia increases KLF8 expression and inhibition of KLF8-reversed hypoxia induces EMT. (A) Western blot analysis of HIF-1 and KLF8 expression in hypoxia at $0,4,8,12$ and $24 \mathrm{~h}$. (B) Analysis of KLF8 mRNA level normalized by GAPDH of SGC7901 cells in hypoxia at 0,4, 8, 12 and $24 \mathrm{~h}$. (C) Immunofluorescence for E-cadherin and fibronectin expression in SGC7901 cells in normoxia, SGC7901/KLF-si and SGC7901/Scr-si cells in hypoxia for 24 h. (D) Morphological changes of SGC7901 cells in normoxia or transfected with KLF-siRNA or Scr-siRNA in hypoxia. Representative images are shown; original magnification, x200. (E) Western blot analysis of epithelial markers (i.e. E-cadherin and keratin) and mesenchymal markers (i.e. fibronectin and vimentin) in SGC7901 cells transfected with the HIF-siRNA or Scr-siRNA as a control in hypoxia. KLF8, Krüppel-like factor 8; EMT, epithelial-mesenchymal transition. 


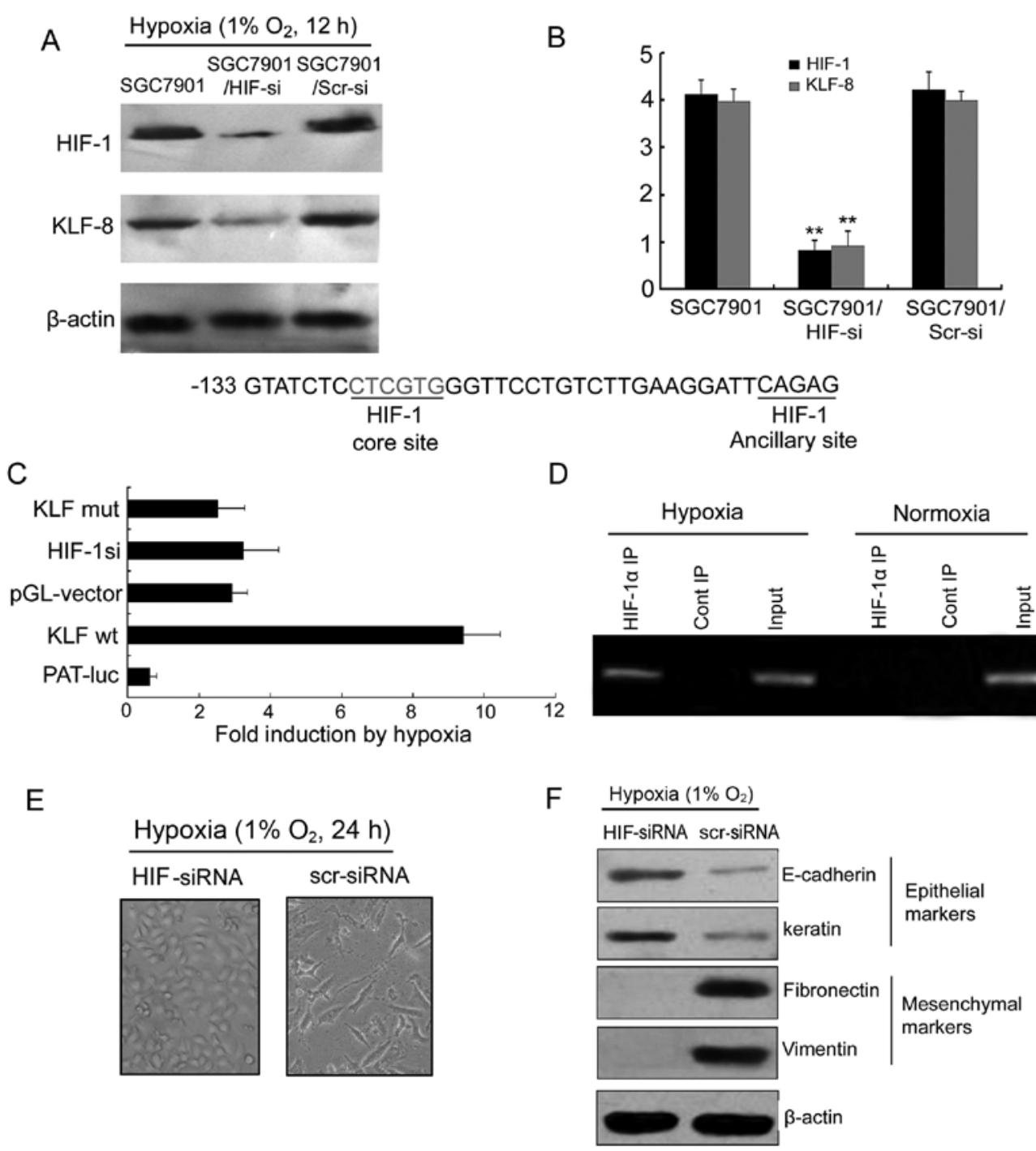

Figure 4. HIF-1 involved in hypoxia-induced KLF8 and EMT. (A) Western blot analysis of HIF-1 and KLF8 expression of SGC7901, SGC7901/HIF-si, SGC7901/Scr-si in hypoxia at $12 \mathrm{~h}$. (B) RT-PCR analysis of HIF-1 and KLF8 expression of SGC7901, SGC7901/HIF-si and SGC7901/Scr-si in hypoxia at $12 \mathrm{~h}$. (C) Dual luciferase reporter gene assay for hypoxia induced KLF8 transcriptional activity. (D) CHIP assay was performed to examine the binding of HIF-1 to KLF8 promoter in normoxia and hypoxia. (E) Morphological assessment of HIF-1 siRNA or scr-siRNA transfected SGC7901 cells under hypoxic condition. (F) Western blot analysis of epithelial markers (i.e., E-cadherin and keratin) and mesenchymal markers (i.e., fibronectin and vimentin) in SGC7901 cells transfected with the HIF-1siRNA or SGC7901 transfected with scr-siRNA as a control. KLF8, Krüppel-like factor 8; EMT, epithelial-mesenchymal transition.

expression of E-cadherin, keratin, fibronectin and vimentin EMT markers. Hypoxia and KLF8-siRNA affected the protein level of E-cadherin and fibronectin, as confirmed by immunofluorescence staining (Fig. 3C). E-cadherin exhibited a strong expression, while fibronectin exhibited a weak expression in the SGC7901 cell line under normoxia. However, under hypoxic conditions $\left(1 \% \mathrm{O}_{2}, 24 \mathrm{~h}\right)$, immunofluorescence staining showed that E-cadherin expression had almost disappeared in SGC7901/Scr-si cells but markedly increased in SGC7901/KLF-si cells. By contrast, fibronectin was increased significantly in SGC7901/Scr-si and hardly observed in SGC7901/KLF-si cells. These findings suggested one of the hallmarks of EMT. To confirm whether EMT can be reversed by KLF-si RNA under hypoxia, we examined several epithelial and mesenchymal markers. As shown in Fig. 3E, SGC7901/ Scr-si cells exhibited an enhanced expression of the fibronectin and vimentin mesenchymal markers, which had almost disappeared for E-cadherin and keratin epithelial marker expression. However, in SGC7901/KLF-si cells, the results were completely reversed compared with SGC7901/Scr-si cells. Consequently, the morphological and protein changes evident in the SGC7901/Scr-si cells in hypoxia indicated that these cells had undergone EMT, a process that can be reversed by KLF8-siRNA.

HIF-1 increases KLF8 expression and transcriptional activity involved in hypoxia-induced EMT. To determine the role of HIF-1 in hypoxia-induced KLF8 expression, HIF-1 siRNA vector was constructed and transfected into the SGC7901 gastric cancer cell line. Western blotting and RT-PCR assays revealed that inhibition of HIF-1 with targeted siRNA could block hypoxia-induced KLF8 expression (Fig. 4A and B). Dual luciferase reporter gene assay showed that luciferase activity transfected with pGL3-KLF8wt was markedly increased compared with the pGL-vector under hypoxia. However, luciferase activity transfected with pGL3-KLF8mut had no 
significant difference compared with the pGL-vector. Thus, it is possible that the HIF-1 binding site is located -140 to -101 from the translation start site of the human KLF8 gene. The possible site contains the HIF-1 core sequence 5'-CTCGTG-3' between -126 to -121 and HIF-1 ancillary sequence 5'-CAG AG-3' (Fig. 4C). Chromatin immunoprecipitation (ChIP) was used to analyze the 1 HRE of KLF8 promoters in SGC7901 tumor cells exposed to hypoxia or normoxia. An obvious band containing the possible binding site in the hypoxic environment but not the normoxic one was observed (Fig. 3D). There was no band in control IgG immunoprecipitates in the hypoxic and normoxic conditions. These results show that the proximal HRE at -133 is the main HIF-1 binding site in the KLF8 promoter.

As shown in Fig. 4E and F, in SGC7901/HIF-si cells, the EMT phenotype was almost completely reversed compared with SGC7901/Scr-si cells. Consequently, protein changes evident in the SGC7901/HIF-si cells in hypoxia suggested that the EMT process can also be reversed by HIF-siRNA.

\section{Discussion}

Gastric cancer is the second leading cause of cancer-related mortalities worldwide $(17,18)$. As symptoms are often absent in the early stages of disease, gastric cancer is usually diagnosed at an advanced stage and there is $<20 \%$ 5-year survival for patients. Gastric cancer deaths results from cancer cell migration and invasion. Therefore, exploring the mechanisms involved is essential in finding a cure. Human KLF8 (ZNF741) was first cloned by PCR from K562 cells, a human hematopoietic cell line (19). KLF8 is broadly expressed in human tissues, with the greatest expression in kidney, heart and placenta. Various KLF8 transcripts have been identified, and the relative levels of the transcript expression appear to be similar to that in various tissues. In a previous study, we confirmed that KLF8 is important in SGC7901 gastric cell progression in vitro and in vivo (12). However, its underlying role in SGC7901 cells with invasion and migration under normoxic or hypoxic conditions have yet to be adequately elucidated. In the present study, we revealed a novel role and mechanisms of KLF8 in the mediation of EMT in gastric cancer and firstly confirmed a novel HRE binding site of HIF-1 in the KLF8 promoter.

We confirmed KLF8 is important in gastric cancer invasion and that the KLF8 siRNA-transfected SGC7901 cell line may decrease gastric cancer cell invasion in vitro and in vivo. In addition, the E-cadherin protein was found to be downregulated, whereas fibronectin was upregulated following infection of the KLF cDNA vector (20). Findings of recent studies have shown that the epithelial cell adhesion molecule E-cadherin is often downregulated during carcinoma progression and metastatic tumor spread (21-24). These observations suggest that KLF8 plays a vital role in EMT in gastric cancer cells. It is well known that the lack of E-cadherin plays an important role in the development of gastric cancer and EMT. Three mechanisms of gene deletion include mutation (25), methylation (26) and E-box in the promoter region $(27,28)$. Results of a previous study have shown that KLF17 is a negative regulation of E-cadherin by binding the E-Box promoter in breast cancer. The association of KLF8 and E-cadherin promoter remains to be investigated (29).
Hypoxia is an important characteristic in the tumor tissue microenvironment and is associated with several pathophysiological processes in tumor progression $(30,31)$. Malignant cancer cells form the tumor chords by colonic proliferation at a diameter of 200-300 $\mu \mathrm{m}$. When the radius of the chords exceeds $300 \mu \mathrm{m}$, the cells in the middle of the chords may undergo hypoxia. However, hypoxia leads to cell progression with a more invasive phenotype. HIF is a transcription factor involved in several biological processes. HIF-1 is able to generate the Warburg effect through enhanced glucose uptake and the downregulation of mitochondrial activities $(32,33)$. Previously, it was suggested that the hypoxic signal can be triggered through HIF, resulting in the occurrence of EMT (34). Tumor growth factor- $\beta$ was initially confirmed as an inducer of EMT in normal mammary epithelial cells and has since been shown to mediate the EMT in tumorigenesis $(35,36)$. Various transcription factors are involved in TGF $\beta$ signals, including ZEB1 and SNAIL (37). Copple (36) suggested that hypoxia stimulates the EMT by a HIF- $1 \alpha$ - and TGF- $\beta$-dependent mechanism during liver fibrosis in hepatocyte. In addition, Shah et al demonstrated SNAIL is associated with breast cancer metastatic progression by inhibiting E-cadherin, which is a hallmark of EMT (23). We employed a KLF8 siRNA to infect SGC7901 in hypoxia to examine the effect of KLF8 silencing on EMT markers and cell morphological changes. It was observed that E-cadherin and keratin epithelial markers were overexpressed while fibronectin and vimentin mesenchymal markers were downregulated in the KLF-si group under hypoxia. Immunofluorescence also confirmed that KLF8 siRNA is able to inhibit the EMT process in hypoxia. These findings suggest that EMT-regulating transcription inhibitors may be crucial in the repression of E-cadherin transcription in gastric tumor. Additionally, KLF8 controls the transcription of E-cadherin, fibronectin, EMT and invasion of its target gene promoters.

Results of previous studies have shown that HIF modulates EMT by regulating the activity of dominant transcription factors including SNAIL, SLUG and ZEB1. EMT has been induced in different cell lines under hypoxia or the continuous overexpression of HIF (38-42). The association between EMT and hypoxia-related transcription factors to EMT processes serve as a molecular explanation for cancer metastasis and hypoxia. Our results have shown that reduced endogenous HIF-1 in SGC7901/HIF-si cells under hypoxia downregulated the KLF8 level, reversed the expression of the epithelial markers and inhibited mesenchymal marker expression. This findings suggests that HIF-siRNA caused the shift of EMT to MET, and this process is accompanied by the significant downregulation of KLF8. Our subsequent results revealed that hypoxia-induced KLF8 was upregulated via a HIF-1-dependent mechanism. There is a possible binding site of HIF-1 at the KLF8 gene promoter. The dual luciferase reporter gene assay results show that hypoxia induced KLF8 promoter activity but was blocked by HIF-1 siRNA. Mutant HRE reporter gene activity decreased. The ChIP assay showed that the proximal HRE at -133 is the main HIF-1 binding site in the KLF8 promoter.

In summary, we have identified KLF8, which was a representative transcriptional factor in the regulation of EMT and SGC7901 gastric cancer cell invasion and migration. The results 
confirmed that KLF8 is a new HIF-1 target gene that may be used to elucidate hypoxia-induced EMT in SGC7901 cells. These results suggest that HIF-1-dependent induction of KLF8 in hypoxia is a promising therapeutic target for gastric cancer or other solid tumors. However, the mechanisms underlying the role of KLF8 in these processes remains to be determined.

\section{Acknowledgements}

This study was supported by the National Nature Science Foundation of China (nos. 81272349 and 81372608).

\section{References}

1. Chaffer CL and Weinberg RA: A perspective on cancer cell metastasis. Science 331: 1559-1564, 2011.

2. Carmeliet P, Dor Y, Herbert JM, et al: Role of HIF-1 $\alpha$ in hypoxiamediated apoptosis, cell proliferation and tumour angiogenesis Nature 394: 485-490, 1998

3. Min JH, Yang H, Ivan M, Gertler F, Kaelin WG Jr and Pavletich NP: Structure of an HIF-1 $\alpha-p V H L$ complex: hydroxyproline recognition in signaling. Science 296: 1886-1889, 2002.

4. Liu L, Sun L, Zhao P, et al: Hypoxia promotes metastasis in human gastric cancer by up-regulating the $67-\mathrm{kDa}$ laminin receptor. Cancer Sci 101: 1653-1660, 2010.

5. Liu L, Zhang H, Sun L, et al: ERK/MAPK activation involves hypoxia-induced MGrl-Ag/37LRP expression and contributes to apoptosis resistance in gastric cancer. Int J Cancer 127: 820-829, 2010.

6. Wang Y, Li Z, Zhang H, et al: HIF-1 $\alpha$ and HIF-2 $\alpha$ correlate with migration and invasion in gastric cancer. Cancer Biol Ther 10 376-382, 2010

7. Liu L, Ning X, Sun L, et al: Hypoxia-inducible factor-1 $\alpha$ contributes to hypoxia-induced chemoresistance in gastric cancer. Cancer Sci 99: 121-128, 2008.

8. Semenza GL: Targeting HIF-1 for cancer therapy. Nat Rev Cancer 3: 721-732, 2003.

9. Lundgren K, Nordenskjöld B and Landberg G: Hypoxia, Snail and incomplete epithelial-mesenchymal transition in breast cancer. Br J Cancer 101: 1769-1781, 2009.

10. Yang MH, Wu MZ, Chiou SH, et al: Direct regulation of TWIST by HIF-1 $\alpha$ promotes metastasis. Nat Cell Biol 10: 295-305, 2008.

11. Yang MH and Wu KJ: TWIST activation by hypoxia inducible factor-1 (HIF-1): implications in metastasis and development. Cell Cycle 7: 2090-2096, 2008.

12. Liu L, Liu N, Xu M, et al: Lentivirus-delivered Krüppel-like factor 8 small interfering RNA inhibits gastric cancer cell growth in vitro and in vivo. Tumour Biol 33: 53-61, 2012.

13. Wang $X$ and Zhao J: KLF8 transcription factor participates in oncogenic transformation. Oncogene 26: 456-461, 2007.

14. Wang X, Zheng M, Liu G, et al: Krüppel-like factor 8 induces epithelial to mesenchymal transition and epithelial cell invasion. Cancer Res 67: 7184-7193, 2007.

15. Fu WJ, Li JC, Wu XY, et al: Small interference RNA targeting Krüppel-like factor 8 inhibits the renal carcinoma 786-0 cells growth in vitro and in vivo. J Cancer Res Clin Oncol 136 $1255-1265,2010$

16. Fink T, Kazlauskas A, Poellinger L, Ebbesen P and Zachar V: Identification of a tightly regulated hypoxia-response element in the promoter of human plasminogen activator inhibitor-1. Blood 99: 2077-2083, 2002.

17. Shah MA and Ajani JA: Gastric cancer - an enigmatic and heterogeneous disease. JAMA 303: 1753-1754, 2010.

18. Jemal A, Bray F, Center MM, Ferlay J, Ward E and Forman D: Global cancer statistics. CA Cancer J Clin 61: 69-90, 2011.

19. Hu JH, Navas P, Cao H, Stamatoyannopoulos G and Song CZ: Systematic RNAi studies on the role of Sp/KLF factors in globin gene expression and erythroid differentiation. J Mol Biol 366: 1064-1073, 2007.

20. Sun Z, Han Q, Zhou N, et al: MicroRNA-9 enhances migration and invasion through KLF17 in hepatocellular carcinoma. Mol Oncol 7: 884-894, 2013.

21. Deep G, Jain AK, Ramteke A, et al: SNAI1 is critical for the aggressiveness of prostate cancer cells with low E-cadherin. Mol Cancer 13: 37, 2014.
22. Chan SW, Kallarakkal TG and Abraham MT: Changed expression of E-cadherin and galectin-9 in oral squamous cell carcinomas but lack of potential as prognostic markers. Asian Pac J Cancer Prev 15: 2145-2152, 2014.

23. Shah P, Gau Y and Sabnis G: Histone deacetylase inhibitor entinostat reverses epithelial to mesenchymal transition of breast cancer cells by reversing the repression of E-cadherin. Breast Cancer Res Treat 143: 99-111, 2014.

24. Wang Y, Dong H, Xu M, et al: 37-kDa laminin receptor precursor promotes lung adenocarcinoma cell invasion and metastasis by epithelial-to-mesenchymal transition. Cancer Gene Ther 21: 150-157, 2014.

25. Machado JC, Soares P, Carneiro F, et al: E-cadherin gene mutations provide a genetic basis for the phenotypic divergence of mixed gastric carcinomas. Lab Invest 79: 459-465, 1999.

26. Leung WK, Yu J, Ng EK, et al: Concurrent hypermethylation of multiple tumor-related genes in gastric carcinoma and adjacent normal tissues. Cancer 91: 2294-2301, 2001.

27. D'souza B and Taylor-Papadimitriou J: Overexpression of ERBB2 in human mammary epithelial cells signals inhibition of transcription of the E-cadherin gene. Proc Natl Acad Sci USA 91: 7202-7206, 1994.

28. Dong C, Wu Y, Wang Y, et al: Interaction with Suv39H1 is critical for Snail-mediated E-cadherin repression in breast cancer. Oncogene 32: 1351-1362, 2013.

29. Gumireddy K, Li A, Gimotty PA, et al: KLF17 is a negative regulator of epithelial-mesenchymal transition and metastasis in breast cancer. Nat Cell Biol 11: 1297-1304, 2009.

30. Muñoz-Nájar UM, Neurath KM, Vumbaca F and Claffey KP: Hypoxia stimulates breast carcinoma cell invasion through MT1-MMP and MMP-2 activation. Oncogene 25: 2379-2392, 2006.

31. McMahon S, Grondin F, McDonald PP, Richard DE and Dubois CM: Hypoxia-enhanced expression of the proprotein convertase furin is mediated by hypoxia-inducible factor-1: impact on the bioactivation of proproteins. J Biol Chem 280: 6561-6569, 2005.

32. Stoeltzing O, McCarty MF, Wey JS, et al: Role of hypoxiainducible factor $1 \alpha$ in gastric cancer cell growth, angiogenesis, and vessel maturation. J Natl Cancer Inst 96: 946-956, 2004.

33. Kong T, Eltzschig HK, Karhausen J, Colgan SP and Shelley CS: Leukocyte adhesion during hypoxia is mediated by HIF-1dependent induction of $\beta_{2}$ integrin gene expression. Proc Natl Acad Sci USA 101: 10440-10445, 2004.

34. Higgins DF, Kimura K, Bernhardt WM, et al: Hypoxia promotes fibrogenesis in vivo via HIF-1 stimulation of epithelial-tomesenchymal transition. J Clin Invest 117: 3810-3820, 2007.

35. Zhou G, Dada LA, Wu M, et al: Hypoxia-induced alveolar epithelial-mesenchymal transition requires mitochondrial ROS and hypoxia-inducible factor 1 . Am J Physiol Lung Cell Mol Physiol 297: L1120-L1130, 2009.

36. Copple BL: Hypoxia stimulates hepatocyte epithelial to mesenchymal transition by hypoxia-inducible factor and transforming growth factor- $\beta$-dependent mechanisms. Liver Int 30: 669-682, 2010.

37. Leivonen SK and Kähäri VM: Transforming growth factor- $\beta$ signaling in cancer invasion and metastasis. Int J Cancer 121: 2119-2124, 2007

38. Shields MA, Dangi-Garimella S, Krantz SB, Bentrem DJ and Munshi HG: Pancreatic cancer cells respond to type I collagen by inducing snail expression to promote membrane type 1 matrix metalloproteinase-dependent collagen invasion. J Biol Chem 286: 10495-10504, 2011.

39. Kang Y and Massagué J: Epithelial-mesenchymal transitions: twist in development and metastasis. Cell 118: 277-279, 2004

40. Aigner K, Dampier B, Descovich L, et al: The transcription factor ZEB1 ( $\delta E F 1)$ promotes tumour cell dedifferentiation by repressing master regulators of epithelial polarity. Oncogene 26: 6979-6988, 2007.

41. Guaita S, Puig I, Franci C, et al: Snail induction of epithelial to mesenchymal transition in tumor cells is accompanied by $M U C 1$ repression and ZEB1 expression. J Biol Chem 277: 39209-39216, 2002.

42. Peinado $\mathrm{H}$ and Cano A: A hypoxic twist in metastasis. Nat Cell Biol 10: 253-254, 2008. 